\title{
In-hospital major arrhythmias, arrhythmic death and resuscitation after successful primary percutaneous intervention for acute transmural infarction: a retrospective single-centre cohort study
}

\author{
Marco Albanese ${ }^{1,3,4^{*}}$ (D) Korhan Alpaslan ${ }^{1}$, Taoufik Ouarrak ${ }^{2}$, Peter Merguet ${ }^{1}$, Steffen Schneider ${ }^{2}$ and Wolfgang Schöls
}

\begin{abstract}
Background: Transmural acute myocardial infarction (AMI) is associated with a high risk for ventricular arrhythmia before, during and after treatment. Consequently, it is recommended that patients diagnosed with transmural AMI be monitored in a cardiac care unit (CCU) so life-threatening arrhythmias can be treated promptly. We examined the incidence and timing of in-hospital malignant ventricular arrhythmias, sudden cardiac or arrhythmic death (SCD/AD) and resuscitation requirements in patients with transmural AMl recovering from percutaneous coronary intervention (PCl) undertaken within $12 \mathrm{~h}$ of symptom onset and without antecedent thrombolysis.
\end{abstract}

Methods: This was a retrospective cohort study using the Duisburg Heart Center (Germany) cardiac patient registry. In total, 975 patients met the inclusion criteria. The composite endpoint was post-PCl ventricular fibrillation or tachycardia, SCD/AD or requirement for resuscitation. We compared the demographic and clinical characteristics of patients who met the composite endpoint with those who did not, recorded the timing of endpoint episodes, and used multivariable logistic regression analysis to identify factors associated with the endpoint criteria.

Results: There was no significant difference in the length of CCU or hospital stay between the groups. In-hospital mortality was $6.5 \%$, and the composite endpoint was met in $7.4 \%$ of cases. Malignant ventricular tachyarrhythmia occurred in $2.8 \%$ of the patients, and SCD/AD occurred in $0.3 \%$ of the cases. There was a biphasic temporal distribution of endpoint events; specifically, 76.7\% occurred < $96 \mathrm{~h}$ after symptom onset, and 12.6\% occurred 240-360 h after symptom onset. Multivariable regression analysis identified positive associations between an endpoint episode and the following: age (odds ratio [OR] 1.03, 95\% confidence interval [CI] 1.01-1.05] per year); left ventricular ejection fraction (LVEF) < 30\% (OR 3.66, 95\% Cl 1.91-6.99); peak serum creatine phosphokinase concentration (OR 1.01, 95\% Cl 1.00-1.02 per $100 \mathrm{U} / \mathrm{dl}$ ); leucocytosis (OR 1.86, 95\% Cl 1.04-3.32), and coronary thrombus (OR 1.85, 95\% Cl 1.04-3.27).

Conclusions: Most post-PCI malignant ventricular arrhythmias, SCD/AD and resuscitation episodes occurred within $96 \mathrm{~h}$ of transmural AMI (76.7\%). A substantial minority (12.6\%) of these events arose 240-360 h after symptom onset. Further study is needed to establish the influence of age, LVEF $<30 \%$, peak serum creatine phosphokinase concentration, leucocytosis and coronary thrombus on post-PCl outcomes after transmural AMI.

Keywords: Myocardial infarction, Arrhythmias, cardiac, Ventricular fibrillation, Tachycardia, ventricular, Resuscitation, Death, sudden, cardiac

\footnotetext{
* Correspondence: m.albanese@hin.ch

${ }^{1}$ Herzzentrum Duisburg, Gerrickstr. 21, D-47137 Duisburg, Germany

${ }^{3}$ Klinik für Kardiologie und Angiologie, Herzzentrum Duisburg, Gerrickstr. 21,

47137 Duisburg, Germany

Full list of author information is available at the end of the article
}

(c) The Author(s). 2018 Open Access This article is distributed under the terms of the Creative Commons Attribution 4.0 International License (http://creativecommons.org/licenses/by/4.0/), which permits unrestricted use, distribution, and

reproduction in any medium, provided you give appropriate credit to the original author(s) and the source, provide a link to the Creative Commons license, and indicate if changes were made. The Creative Commons Public Domain Dedication waiver (http://creativecommons.org/publicdomain/zero/1.0/) applies to the data made available in this article, unless otherwise stated. 


\section{Background}

Transmural acute myocardial infarction (AMI) is associated with a high risk for life-threatening arrhythmias in the early phase of an ischaemic event [1]. Current guidelines suggest that patients with transmural AMI be admitted to the intensive cardiac care unit (CCU) for 24-48 h after symptom onset [2]. Admission to the CCU allows prompt resuscitation in the event of cardiac arrest but has a substantial impact on treatment costs [3]. Although several models exist for predicting early complications in AMI, major arrhythmias have not been addressed in these models [4].

Mechanical reperfusion has become the treatment of choice for transmural AMI. Compared with thrombolysis, reperfusion has been shown to achieve higher rates of the best grade of coronary flow (Thrombolysis in Myocardial Infarction [TIMI] grade 3) and to reduce the incidence of recurrent ischaemia and reinfarction as well as infarct size, thus preserving left ventricular ejection fraction (LVEF) [57]. Nonetheless, there are limited data on the potential therapeutic benefit of primary percutaneous coronary intervention (PCI) in terms of the incidence of in-hospital malignant arrhythmias in patients with acute ST-elevation MI (STEMI). The incidence of in-hospital ventricular arrhythmias was not reported in large clinical trials, such as PRAGUE-2 or DANAMI-2 [7, 8]. A meta-analysis of large clinical trials indicates that the incidence of ventricular fibrillation (VF) appears to be lower in patients treated with PCI compared with that in patients treated with thrombolysis [1]. Several single-centre reports have yielded conflicting results on the incidence of in-hospital arrhythmias after primary PCI for acute STEMI. According to Giglioli and colleagues, no in-hospital life-threatening arrhythmias occurred after PCI; most life-threatening arrhythmias arose in the catheterization laboratory $[9,10]$. Similarly, in a Swiss study, most arrhythmias occurred in the catheterization laboratory, nearly all within $24 \mathrm{~h}$; a report from Germany revealed that $90 \%$ of fatal events occurred within the first $48 \mathrm{~h}[11,12]$.

In our clinical practice, we were struck by the sudden cardiac death (SCD) of a 40-year-old patient 96 h after symptom onset in an otherwise apparently uncomplicated anterior STEMI. This patient satisfied none of the predictive factors, such as LVEF $<40 \%$, commonly used for risk stratification. To better understand the timing of and risk factors for major arrhythmias in a current patient population treated by PCI, we retrospectively analysed the data of 975 patients treated at our tertiary care centre. Our analysis differed from that in the Swiss and German single-centre studies referred to above. As the analysis was limited to patients treated by primary PCI within $12 \mathrm{~h}$ of symptom onset and without antecedent thrombolysis, our cohort was a homogenous patient population that had only been exposed to the therapeutic consequences of PCI. In addition, we defined transmural AMI using angiographic evidence of an occluded vessel to ensure identification of transmural infarctions without ST-elevation on the surface electrocardiogram (ECG). A STEMI and this well-defined non-ST-elevation myocardial infarction (NSTEMI) subgroup were thus included in our cohort, as they represent a common pathoanatomical substrate associated with an increased risk of fatal events. We sought to establish whether $48 \mathrm{~h}$ of monitoring was sufficient to prevent fatal events and to determine whether clinical predictors of the occurrence of fatal events in our patient population differed from those described in previous publications.

Our primary objective was to determine the timing and total burden of in-hospital major arrhythmias after successful primary PCI for transmural AMI undertaken within $12 \mathrm{~h}$ of symptom onset without antecedent thrombolysis. The secondary objective was to identify clinical predictors associated with the occurrence of the combined endpoint of ventricular tachycardia (VT), SCD or arrhythmic death (SCD/AD) and resuscitation in the early phase of acute transmural infarction.

\section{Methods \\ Study patients and data collection}

This was a retrospective cohort study using our institution's cardiac patient registry, into which data are input on discharge from hospital or after in-hospital death. Data collection began on January 1, 2005, and ended on May 17,2011 . We selected patients with symptom onset $<12 \mathrm{~h}$ before performance of PCI and evidence of transmural AMI as reflected by ST-segment elevation in at least two contiguous leads or the presence of left bundle branch block (LBBB) on the surface ECG or by angiographic evidence of AMI as determined by the presence of an occluded vessel. This definition of transmural AMI was chosen to ensure that transmural infarctions without ST-elevation were not missed; for example, left circumflex or right coronary artery occlusion without ST-elevation in the appropriate leads. Patients were excluded if they had undergone antecedent thrombolysis, if they were not treated by primary PCI, if primary PCI was not successful or if coronary artery bypass surgery was performed. Cardiogenic shock was not an exclusion criterion. The selection of patients in the cohort is shown in Fig. 1.

All patients were treated by a single group of cardiologists at the Duisburg Heart Centre, Duisburg, Germany, a tertiary care centre. Approximately $70 \%$ of the patients were admitted directly, with the remainder transferred from primary or secondary care centres where PCI was not available.

\section{Catheterization and percutaneous coronary intervention}

Patients were treated with an oral loading of $300 \mathrm{mg}$ clopidogrel. Further medical therapy was given, and the choice of bare metal or drug-eluting stents was made according 


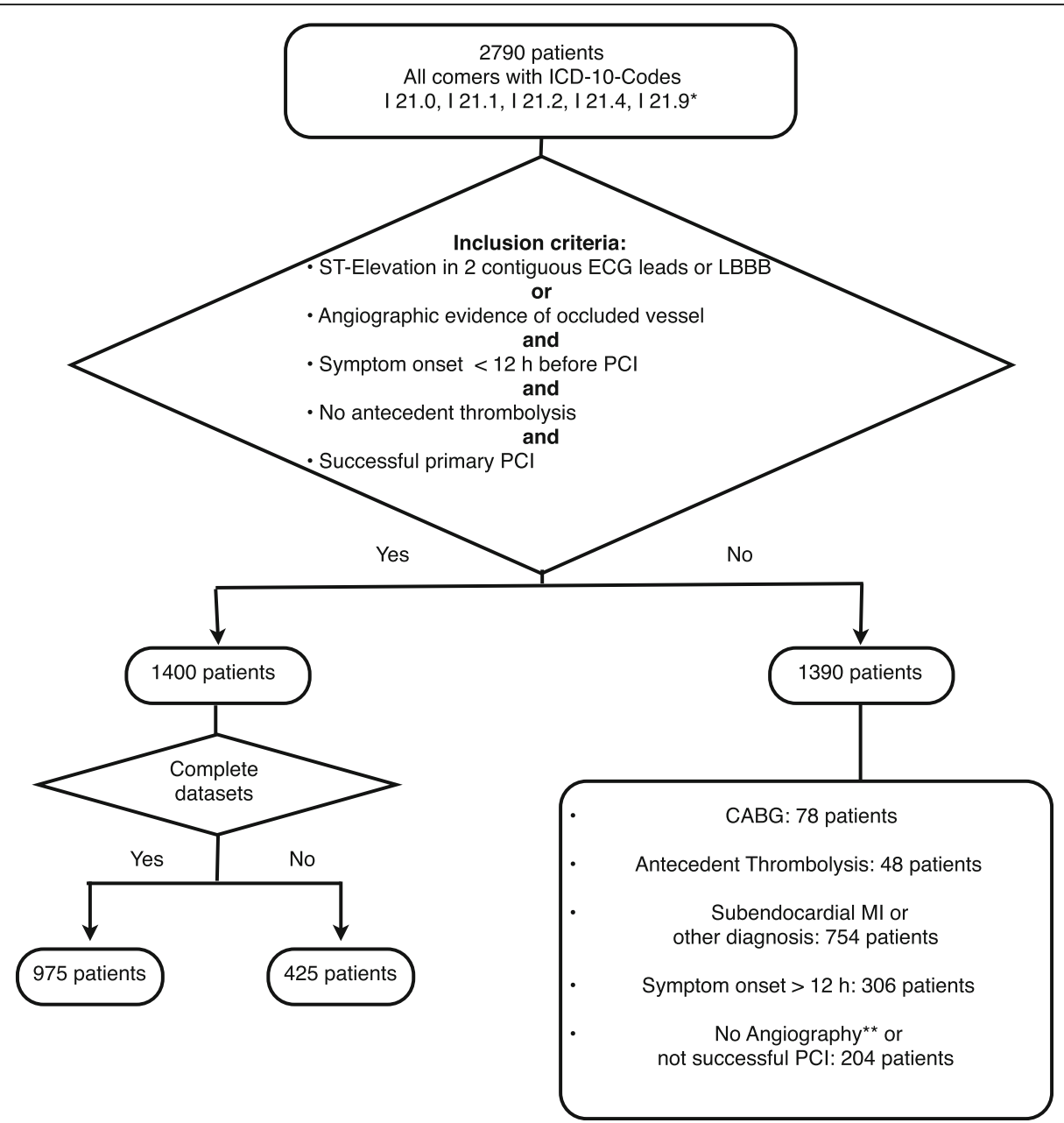

Fig. 1 Study flow chart. * International Classification of Disease (ICD)-10 codes: | 21.0, acute transmural anterior myocardial infarction; | 21.1, acute transmural inferior myocardial infarction; I 21.2, acute transmural myocardial infarction of other sites; | 21.3, acute transmural myocardial infarction of unspecified site; I 21.4, acute subendocardial myocardial infarction; I 21.9, acute myocardial infarction, not precisely specified. ** no consent, death beforehand or contraindications. Other abbreviations: ECG, electrocardiogram; LBBB, left bundle branch block; PCl, percutaneous coronary intervention; $C A B G$, coronary artery bypass grafting

to current national guidelines. Treatment with glycoprotein IIb/IIIa inhibitors or bivalirudin was performed at the cardiologist's discretion. In most cases, only culprit lesions were treated by primary PCI, i.e., only the lesion of the infarct-related artery (IRA) was treated directly by angioplasty and stent delivery. Patients with cardiogenic shock instead underwent the most complete revascularization possible; specifically, all stenosed segments, including those vessels that were not the IRA, were treated by multiple PCIs during the acute intervention. Angioplasty success was defined as $<50 \%$ stenosis and a TIMI flow of grade 2 or 3; coronary thrombus was identified on angiography and by TIMI flow.

\section{Endpoints and definitions}

The primary combined endpoints were the occurrence and timing of in-hospital ventricular arrhythmia, $\mathrm{SCD} / \mathrm{AD}$ or resuscitation after primary PCI. All endpoints were either monitored or witnessed by experienced personnel or were ascertained by autopsy. Malignant ventricular arrhythmia or malignant tachyarrhythmia included VF or VT with haemodynamic compromise, and SCD was an unexpected death resulting from heart disease occurring within $1 \mathrm{~h}$ of symptom onset or unwitnessed overnight. Arrhythmic death was an unexpected death resulting from arrhythmia other than SCD or VT, i.e., complete heart block (CHB), pulseless electrical activity (PEA) or asystole. Resuscitation was defined as cardiocirculatory arrest followed by basic/ advanced life support according to International Liaison Committee on Resuscitation (ILCOR) or American Heart Association (AHA) guidelines. Cardiac arrest was assessed by experienced medical and paramedic personnel based on telemetry detection of heart rhythm, blood pressure monitoring and pulse oxymetry or, in late events, on clinical 
evaluation. Any episodes before or during catheterization were not taken into account. Telephone follow-up was undertaken for the $7 \%$ of patients who had been transferred to other hospitals after primary PCI. No other follow-up was performed in this study, which focused on in-hospital events and the possible prevention of such events.

According to hospital policy, all patients were transferred to the CCU after PCI and were then continuously monitored for complex arrhythmia for a minimum of 48 h. Telemetry was used to monitor high-risk patients after transfer to the ward. Endpoints occurring throughout the entire hospital stay were analysed, and an autopsy was performed in unclear cases.

Normal left ventricular function was defined as an LVEF $>50 \%$, slightly impaired function was defined as $40-50 \%$, moderately impaired function was defined as $30-40 \%$ and severely impaired function was defined as $<30 \%$. Contrast ventriculography or echocardiography was used to estimate LVEF immediately after admission.

\section{Statistical analysis}

Absolute numbers and proportions (expressed as percentages) are used to describe the patient population. Medians (with quartiles) or means (with standard deviations) were calculated as appropriate. Categorical values were compared using the chi-square test, and continuous variables were compared using the two-tailed Wilcoxon rank sum test.

Multivariable logistic regression analysis was performed to identify independent predictors of the combined endpoint. The regression analysis took into account all events recorded. All baseline parameters previously reported to be associated with a higher risk for acute coronary syndrome were used as independent parameters in the model (Table 2) $[4,5] . P$ values $<0.05$ were considered statistically significant. All $p$ values were results of two-tailed tests. All analyses were performed using the SAS statistics programme (version 9.2; SAS Institute Inc., Cary, NC).

\section{Results}

\section{Patient and treatment characteristics}

The demographic and clinical characteristics of the patient population are shown in Table 1; the majority underwent $\mathrm{PCI}<4 \mathrm{~h}$ after symptom onset $(89.3 \%)$, and one or more stents were deployed in $98.7 \%$ of the cases (drug-eluting stents were deployed in $12.6 \%$ of the cases). Left ventricular function was severely impaired in 9.3\% of the patient population. The mean duration of hospitalization was $10 \mathrm{~d}$ (range 7-35 d). Length of hospital stay was not associated with the occurrence of the combined endpoint. There was no statistically significant difference in the duration of CCU stay or of telemetric monitoring between patients who did or did not meet the composite endpoint.
The combined endpoint was observed in $7.4 \%$ of patients. PEA and VT were the most common malignant arrhythmias. Resuscitation was undertaken in $7.1 \%$ of the patients. By analysing the patient population according to the presence or absence of the combined endpoint, we observed the following. Patients who met the endpoint criteria were older, more frequently had a history of previous MI and peripheral arterial disease, more often took aspirin, and were more frequently diagnosed with anterior STEMI, atrial fibrillation or LBBB on admission. Serum troponin and creatine phosphokinase (CK) concentrations were almost doubled, and a higher proportion had an elevated white blood cell count (WBC) compared with those who did not meet the composite endpoint.

During coronary intervention, patients who subsequently met the composite endpoints were found to have a more extensive coronary thrombus, and there was a tendency towards more frequent use of glycoprotein IIb/IIIa inhibitors. More patients with severely impaired LVEF $(<30 \%)$ or who required vasopressor support subsequently met the combined endpoint. There was no difference in coronary status or time to reperfusion between the two groups. Although the difference in stent deployment rate was statistically significant $(P$ $<0.05$ ), it was not considered relevant because of the small difference in absolute numbers (97.2 vs 99.5\%).

Descriptive analysis of the data showed that $27.5 \%$ of the patients who met the endpoint underwent early perfusion (<90 $\mathrm{min}$ ), 64.9\% underwent intermediate perfusion $(1.5-4 \mathrm{~h})$, and $7.6 \%$ underwent late reperfusion $(>4 \mathrm{~h})$. There was no significant difference in the proportions who developed VT (21.6, 18.6 and $18.6 \%$, respectively) or $\operatorname{AD}(32.5,32.2$ and $41.9 \%$, respectively) or who required resuscitation (45.9, 49.2 and $41.9 \%$, respectively).

\section{Predictors of the composite endpoint}

Of the 12 original candidate variables, five remained statistically significant in the multivariate analysis and formed a final set of predictor variables (Table 2). These variables were age, $\mathrm{WBC}>10,000 / 1$, coronary thrombus, LVEF $<30 \%$ and peak serum CK concentration. The presence of atrial fibrillation was not a significant predictor $(P=0.051)$.

\section{Temporal distribution of endpoints and correlation with endpoint predictors}

We observed a biphasic distribution of events: a total of $62.7 \%$ of the combined endpoints occurred within the first $48 \mathrm{~h}$ of symptom onset, and a total of $76.7 \%$ were recorded within $96 \mathrm{~h}$ of symptom onset. Between 240 and $360 \mathrm{~h}$ following symptom onset, the incidence of the combined endpoint was $12.0 \%$. A smaller number of endpoint events occurred later in the clinical course (Fig. 2). 
Table 1 Baseline demographic and clinical characteristics of patients included in the study

\begin{tabular}{|c|c|c|c|c|}
\hline & Total & Patients with composite endpoint & Patients without composite endpoint & $P$-value \\
\hline \multicolumn{5}{|l|}{ Demographics } \\
\hline Total, \% & 975 & $72(7.4 \%)$ & $903(92.6 \%)$ & \\
\hline Female, \% & $29.2 \%(285 / 975)$ & $33.3 \%(24 / 72)$ & $28.9 \%(261 / 903)$ & 0.45 \\
\hline Age, (years) & $62(52-73)$ & $68(53-78)$ & $62(52-72)$ & $<0.05$ \\
\hline \multicolumn{5}{|l|}{ Clinical presentation } \\
\hline $\mathrm{BMI}, \mathrm{kg} / \mathrm{m}^{2}$ & $27(25-30)$ & $26(25-29)$ & $28(25-30)$ & $<0.03$ \\
\hline Adiposity (BMI > 30), \% & $24.0 \%(220 / 916)$ & $12.7 \%(8 / 63)$ & $24.9 \%(212 / 853)$ & $<0.05$ \\
\hline \multicolumn{5}{|l|}{ Medical history } \\
\hline Previous Ml, \% & $14.0 \%(135 / 961)$ & $24.3 \%(17 / 70)$ & $13.2 \%(118 / 891)$ & $<0.05$ \\
\hline Previous $\mathrm{PCl}, \%$ & $12.5 \%(120 / 960)$ & $14.3 \%(10 / 70)$ & $12.4 \%(110 / 890)$ & 0.64 \\
\hline Previous CABG, \% & $3.3 \%(32 / 962)$ & $7.1 \%(5 / 70)$ & $3.0 \%(27 / 892)$ & 0.06 \\
\hline Previous stroke, \% & $3.0 \%(29 / 963)$ & $4.3 \%(3 / 70)$ & $2.9 \%(26 / 893)$ & 0.52 \\
\hline Hypertension, \% & $71.4 \%(688 / 964)$ & $70.4 \%(50 / 71)$ & $71.4 \%(638 / 893)$ & 0.85 \\
\hline Hypercholesterolemia, \% & $74.9 \%(719 / 960)$ & $59.7 \%(43 / 72)$ & $76.1 \%(676 / 888)$ & $<0.01$ \\
\hline Diabetes mellitus, \% & $24.0 \%(234 / 975)$ & $29.1 \%(21 / 72)$ & $23.5 \%(213 / 903)$ & 0.27 \\
\hline Current smoker, \% & $46.9 \%(449 / 958)$ & $31.9 \%(22 / 69)$ & $48.0 \%(427 / 889)$ & $<0.01$ \\
\hline PAD, $\%$ & $5.7 \%(54 / 946)$ & $13.0 \%(9 / 69)$ & $5.1 \%(45 / 877)$ & $<0.01$ \\
\hline Aspirin on admission, \% & $23.7 \%(221 / 957)$ & $37.1 \%(26 / 70)$ & $22.0 \%(195 / 887)$ & $<0.01$ \\
\hline \multicolumn{5}{|l|}{ Laboratory on admission } \\
\hline Potassium, mmol/L & $4.0(3.7-4.3)$ & $4.2(3.8-4.7)$ & $4.0(3.7-4.3)$ & $<0.05$ \\
\hline Creatinine, mg/dl & $0.9(0.8-1.1)$ & $1.1(0.9-1.4)$ & $0.9(0.8-1.1)$ & $<0.0001$ \\
\hline Elevated CRP ( $\geq 5 \times), \%$ & $10.3 \%(76 / 741)$ & $14.3 \%(8 / 56)$ & $9.9 \%(68 / 685)$ & 0.30 \\
\hline WBC > $10.000 \mu \mathrm{l}, \%$ & $57.0 \%(547 / 960)$ & $72.0 \%(52 / 72)$ & $55.7 \%(495 / 885)$ & $<0.01$ \\
\hline $\mathrm{HbA} 1 \mathrm{c}, \%$ & $8.4 \pm 7.5$ & $10.4 \pm 13.8$ & $8.1 \pm 6.4$ & 0.9 \\
\hline \multicolumn{5}{|l|}{ ECG on admission } \\
\hline anterior STEMI, \% & $39.5 \%(383 / 970)$ & $55.6 \%(40 / 72)$ & $38.5 \%(343 / 891)$ & $<0.05$ \\
\hline$A F, \%$ & $4.4 \%(42 / 968)$ & $10.3 \%(7 / 71)$ & $3.9 \%(35 / 890)$ & $<0.01$ \\
\hline LBBB, \% & $1.8 \%(17 / 965)$ & $5.5 \%(4 / 72)$ & $1.5 \%(13 / 888)$ & $<0.05$ \\
\hline AV block, \% & $3.9 \%(38 / 968)$ & $4.1 \%(3 / 72)$ & $3.9 \%(35 / 820)$ & 0.97 \\
\hline \multicolumn{5}{|l|}{ Coronary angiogram and intervention } \\
\hline Three-vessel disease, \% & $27.3 \%(266 / 975)$ & $31.9 \%(23 / 72)$ & $26.9 \%(243 / 903)$ & 0.36 \\
\hline Time to reperfusion $<4$ h, $\%$ & $89.3 \%(868 / 972)$ & $92.4 \%(67 / 72)$ & $89.6 \%(795 / 893)$ & 0.35 \\
\hline Coronary thrombus, \% & $19.2 \%(187 / 973)$ & $33.3 \%(24 / 72)$ & $18.2 \%(163 / 894)$ & $<0.01$ \\
\hline Stent implantation, \% & $98.7 \%(956 / 969)$ & $97.2 \%(70 / 72)$ & $99.5 \%(886 / 890)$ & $<0.05$ \\
\hline GPlla-IIIb antagonist, \% & $2.9 \%(28 / 965)$ & $6.9 \%(5 / 72)$ & $2.6 \%(23 / 887)$ & 0.05 \\
\hline Bivalirudin, \% & $15.0 \%(145 / 968)$ & $20.3 \%(15 / 72)$ & $14.5 \%(130 / 889)$ & 0.17 \\
\hline \multicolumn{5}{|l|}{ Clinical course } \\
\hline CCU stay, h & $46(32-54)$ & $49(20-169)$ & $46(33-52)$ & 0.17 \\
\hline Routine hospital ward stay, h & $150(120-193)$ & $170(105-200)$ & $150(120-193)$ & 0.60 \\
\hline Telemetric monitoring, $\mathrm{h}$ & $47(33-66)$ & $50(13-192)$ & $47(33-58)$ & 0.26 \\
\hline Use of vasopressors, \% & $12.5 \%(122 / 975)$ & $83.5 \%(60 / 72)$ & $6.9 \%(62 / 896)$ & $<0.0001$ \\
\hline Maximum troponin value, $U / L$ & $3.4(1.5-6.4)$ & $5.9(1.7-14.1)$ & $3.3(1.5-6.1)$ & $<0.01$ \\
\hline Maximum CK value, mg/dl & $1413(549-2822)$ & $2178(609-4513)$ & $1351(545-2706)$ & $<0.01$ \\
\hline
\end{tabular}


Table 1 Baseline demographic and clinical characteristics of patients included in the study (Continued)

\begin{tabular}{lllll}
\hline & Total & Patients with composite endpoint & Patients without composite endpoint & $P$-value \\
\hline EF $<30 \%, \%$ & $9.3 \%(80 / 857)$ & $43.4 \%(23 / 53)$ & $7.1 \%(57 / 804)$ & $<0.0001$ \\
Death & $6.5 \%(63 / 974)$ & $72.2 \%(52 / 72)$ & $1.2 \%(11 / 896)$ & $<0.0001$ \\
Cardiac death, \% of total & $88.9 \%(56 / 63)$ & $96.2 \%(50 / 52)$ & $54.5 \%(6 / 11)$ & $<0.001$ \\
\hline
\end{tabular}

Troponin was assayed as troponin $\mathrm{T}$ (normal $<0.01 \mathrm{U} / \mathrm{L})$

Abbreviations: $B M I$ body mass index, $M I$ myocardial infarction, $P C l$ percutaneous coronary intervention, $C A B G$ coronary artery bypass grafting, $P A D$ peripheral artery disease, CRP C-reactive protein, WBC white blood cell count, Hba1c glycated hemoglobin, STEMI ST-elevation MI, AF atrial fibrillation, $L B B B$ left bundle branch block, $A V$ atrioventricular, GP glycoprotein, CCU coronary care unit, CK creatine phosphokinase (normal $<250 \mathrm{mg} / \mathrm{dl}$ ), $E F$ ejection fraction

Specifically, $12.7 \%$ of malignant ventricular arrhythmias and $21.6 \%$ of resuscitations occurred within the first $48 \mathrm{~h}$ of symptom onset. In addition, $13.4 \%$ of VT/VF and $31.7 \%$ of resuscitations were recorded within $96 \mathrm{~h}$ of symptom onset. Between 240 and $360 \mathrm{~h}$ following symptom onset, the incidence of ventricular arrhythmia was $1.4 \%$ and that of resuscitation was $4.2 \%$. In the first $48 \mathrm{~h}$ after AMI, $25.4 \%$ of the SCD/AD episodes were observed, and a total of $31.7 \%$ occurred within $96 \mathrm{~h}$ of symptom onset. The incidence of SCD/AD between 240 and $360 \mathrm{~h}$ following symptom onset was $7 \%$. The exact timing of events is shown in Table 3 (supplemental data).

Due to the low number of events, it was not possible to calculate a risk score. Therefore, we analysed the data for the presence of predictor variables in patients with endpoints that occurred $>48 \mathrm{~h}$ after admission. In $90.0 \%$ of the patients with endpoints $>48 \mathrm{~h}$ after admission, a minimum of two or more predictors was present (data not shown).

\section{Secondary outcomes}

Total in-hospital mortality was $6.5 \%$. Most of the deaths were from a cardiac cause $(88.9 \%)$ and $77.0 \%$ were due to arrhythmia. A minority of cardiac deaths (8.7\%) occurred unobserved late in the clinical course $(>96 \mathrm{~h}$ after admission). Patients experiencing sustained VT or VF had in-hospital mortality rates of 15.4 and $93.3 \%$, respectively. Only $0.8 \%$ developed CHB after successful PCI. Among the patients who developed PEA, VF, CHB or asystole, cardiogenic shock was present in $86.7 \%$ of patients with PEA and 53.3\% of patients with VF (Table 4).

\section{Discussion}

\section{Key findings}

The primary objective of this study was to determine the total burden and timing of in-hospital major arrhythmias and of the combined endpoint of ventricular arrhythmia, SCD/AD or resuscitation after successful primary PCI for transmural AMI within $12 \mathrm{~h}$ of symptom onset. Malignant ventricular tachyarrhythmia occurred in $2.8 \%$ of the patients, bradyarrhythmia occurred in $1.1 \%$, PEA occurred in $2.6 \%$, asystole occurred in $0.8 \%$ and SCD occurred in $0.3 \%$. The combined endpoint was met in $7.4 \%$ of patients. We observed a biphasic distribution of events, with $76.7 \%$ of endpoints occurring within $96 \mathrm{~h}$ of symptom onset and 12.6\% occurring 240-360 d after AMI. Occasional events were, however, observed throughout the hospital stay.

Table 2 Predictors of the composite endpoint in patients with acute myocardial infarction (multivariate analysis)

\begin{tabular}{llll}
\hline & $B$ Coefficient & $P$ value & Odds ratio (95\% Cl) \\
\hline Female & 0.3059 & 0.6699 & $0.878(0.48-1.59)$ \\
Age, years & 0.0118 & 0.008 & $1.032(1.008-1.05)$ \\
PAD & 0.4613 & 0.4581 & $1.408(0.57-3.47)$ \\
anterior STEMI, \% & 0.2774 & 0.4000 & $1.263(0.73-2.17)$ \\
AF, \% & 0.4641 & 0.0514 & $2.470(0.99-6.13)$ \\
LBBB, \% & 0.7615 & 0.3608 & $2.006(0.45-8.92)$ \\
Coronary thrombus, \% & 0.2909 & 0.0339 & $1.853(1.04-3.27)$ \\
EF $<30 \%$ & 0.3307 & $<0.0001$ & $3.656(1.91-6.99)$ \\
Creatinine, mg/dl & 0.0037 & 0.9077 & $1.000(0.99-1.00)$ \\
WBC $>10.000 \mu l$ & 0.0118 & 0.0354 & $1.863(1.04-3.32)$ \\
Maximum troponin value, U/L & 0.0009 & 0.7892 & $1.000(0.99-1.00)$ \\
Maximum CK value, $100 \mathrm{U} / \mathrm{dl}$ & 0.0032 & $<0.0001$ & $1.014(1.00-1.02)$ \\
\hline
\end{tabular}

Abbreviations: $C l$ confidence intervals, $P A D$ peripheral artery disease, STEMI ST-elevation myocardial infarction, $A F$ atrial fibrillation, $L B B B$ left bundle brunch block, $E F$ ejection fraction, WBC white blood cell count, $C K$ creatine phosphokinase 
- Combined endpoint

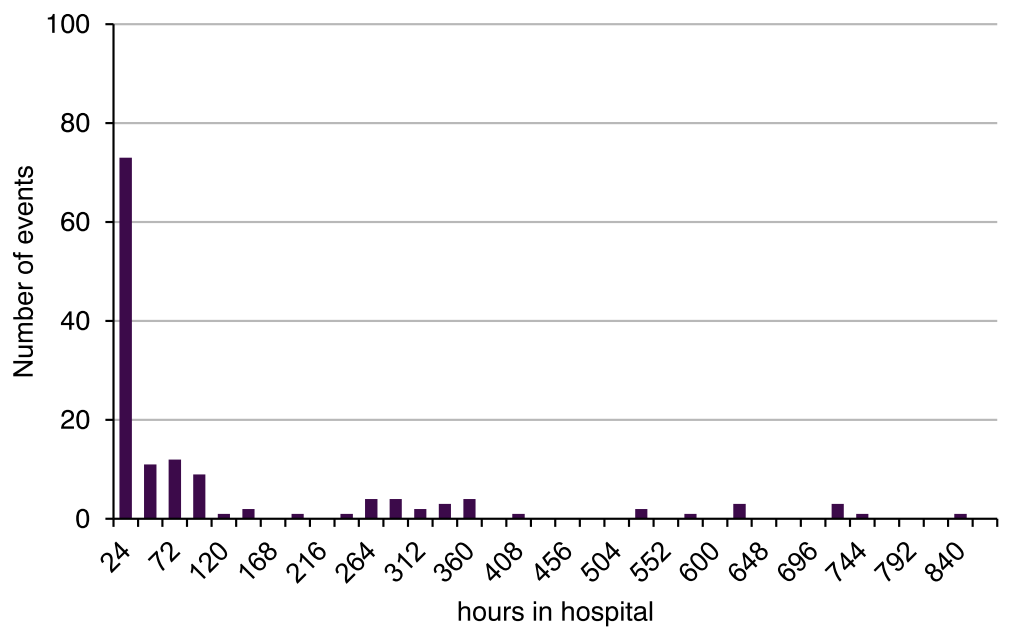

Fig. 2 Distribution of time of events of the combined endpoint in transmural AMl after reperfusion by primary PCI without antecedent thrombolysis. The combined endpoint comprises VT, resuscitation and SCD/arrhythmic death. Multiple events per patient were possible: $24 \mathrm{~h}: 73 ; 48 \mathrm{~h}: 16 ; 72 \mathrm{h:} 11$; 96 h: 9; 120 h: 1; 144 h: 2; 168 h: 0; 192 h: 1; 216 h: 0; 240 h: 1; 264 h: 4; 288 h: 4; 312 h: 2; 336 h: 3; 360 h: 4; 384 h: 0; 408 h: 1; 432 h: 0; 456 h: 0; 480 h: 0; 504 h: 0; 528 h: 1; 552 h: 0; 576 h: 1; 600 h: 0; 624 h: 3; 648 h: 0; 672 h: 0; 696 h: 0; 720 h: 3; 744 h: 1; 768 h: 0; 792 h: 0; 816 h: 0; 840 h: 1

The secondary objective was to identify clinical predictors associated with the occurrence of the combined endpoint, ventricular arrhythmia, SCD/AD or resuscitation in the early phase of acute transmural infarction. Due to the low number of events, we were unable to reliably identify clinical predictors but observed positive associations between the combined endpoint and age, severely impaired LVEF, peak serum CK concentration, leucocytosis and coronary thrombus in the multivariable regression analysis.

\section{Comparison with other studies}

It is difficult to compare our findings with other studies on PCI, which were designed differently and had different endpoints. In some studies, more time elapsed between symptom onset and PCI than in our cohort. In other studies, the occurrence of endpoints before and during cardiac catheterization were taken into account, and the length of observation time and the differentiation of arrhythmias were not the same in all studies.

Table 3 Occurrence of the specific components of the combined endpoint in time

\begin{tabular}{|c|c|c|c|c|c|c|c|c|c|c|}
\hline Adverse event & $24 \mathrm{~h}$ & $48 \mathrm{~h}$ & $72 \mathrm{~h}$ & $96 \mathrm{~h}$ & $120 \mathrm{~h}$ & $144 \mathrm{~h}$ & $168 \mathrm{~h}$ & $192 \mathrm{~h}$ & $216 \mathrm{~h}$ & $240 \mathrm{~h}$ \\
\hline VTNF & 18 & & 1 & & & & & & & \\
\hline$S C D / A D$ & 25 & 11 & 5 & 4 & 1 & 1 & & & & 1 \\
\hline Resuscitation & 30 & 5 & 5 & 5 & & 1 & & 1 & & \\
\hline Adverse event & $264 \mathrm{~h}$ & $288 \mathrm{~h}$ & $312 \mathrm{~h}$ & $336 \mathrm{~h}$ & $360 \mathrm{~h}$ & $384 \mathrm{~h}$ & $408 \mathrm{~h}$ & $432 \mathrm{~h}$ & $456 h$ & $480 \mathrm{~h}$ \\
\hline VTNF & & 1 & & 1 & & & 1 & & & \\
\hline$S C D / A D$ & 3 & 1 & 2 & & 3 & & & & & \\
\hline Resuscitation & 1 & 2 & & 2 & 1 & & & & & \\
\hline Adverse event & $504 \mathrm{~h}$ & $528 \mathrm{~h}$ & $552 \mathrm{~h}$ & $576 \mathrm{~h}$ & $600 \mathrm{~h}$ & $624 h$ & $648 \mathrm{~h}$ & $672 \mathrm{~h}$ & $696 h$ & $720 h$ \\
\hline VTNF & & & & & & 1 & & & & 1 \\
\hline$S C D / A D$ & & 1 & & 1 & & 1 & & & & \\
\hline Resuscitation & & & & & & 1 & & & & 2 \\
\hline Adverse event & $744 \mathrm{~h}$ & $768 \mathrm{~h}$ & $792 \mathrm{~h}$ & $816 \mathrm{~h}$ & $840 \mathrm{~h}$ & & & & & \\
\hline \multicolumn{11}{|l|}{ VTNF } \\
\hline$S C D / A D$ & 1 & & & & 1 & & & & & \\
\hline Resuscitation & & & & & & & & & & \\
\hline
\end{tabular}

Depicted are absolute numbers and multiple events per patient were possible. A total of 142 events divided into specfic events as follows: a) Ventricular tachycardia (VT) 24 events b) SCD/arrhythmic death 62 events c) Resuscitation 56 events 
Table 4 In-hospital arrhythmias: relationship to cardiogenic shock and relative mortality

\begin{tabular}{|c|c|c|c|}
\hline Malignant arrhythmia & $\%$ of total ${ }^{a}$ & Cardiogenic shock $^{\mathrm{b}}$ & Mortality $^{\mathrm{b}}$ \\
\hline \multicolumn{4}{|l|}{ Tachyarrhythmia } \\
\hline sustained VT, \% & $1.3 \%$ & $15.4 \%$ & $15.4 \%$ \\
\hline$V F, \%$ & $1.5 \%$ & $53.3 \%$ & $93.3 \%$ \\
\hline \multicolumn{4}{|l|}{ Bradyarrhythmia } \\
\hline $\mathrm{CHB}, \%$ & $0.8 \%$ & $37.5 \%$ & $62.5 \%$ \\
\hline Bradyarrhythmia absoluta, \% & $0.2 \%$ & $0 \%$ & $0 \%$ \\
\hline Sinus arrest, & $0.1 \%$ & $0 \%$ & $0 \%$ \\
\hline$S C D, \%$ & $0.3 \%$ & $0 \%$ & $100 \%$ \\
\hline PEA, \% & $2.6 \%$ & $86.7 \%$ & $100 \%$ \\
\hline Asystole, \% & $0.8 \%$ & $53.8 \%$ & $84.6 \%$ \\
\hline
\end{tabular}

$V T$ ventricular tachycardia, $V F$ ventricular fibrillation, $C H B$ complete heart block, $S C D$ sudden cardiac death, $P E A$ pulseless electrical activity

${ }^{a}$ with respect to entire study population

${ }^{\mathrm{b}}$ with respect to cases of VT, VF, CHB, Bradyarrhythmia absoluta, sinus arrest, SCD, PEA, Asystole

The studies were, however, broadly comparable regarding study populations, concomitant medications, techniques and stents used.

The incidences of sustained VT or VF and SCD in our registry were 2.8 and $0.3 \%$, respectively. The total burden of in-hospital malignant ventricular arrhythmias in our registry was therefore similar to or lower than that described in other reports of transmural AMI treated by primary PCI. In a similarly designed study by Giglioli et al., only episodes of VF were recorded, which occurred in $0.6 \%$ of patients after cardiac catheterization; however, the absence of reports on other endpoints makes a direct comparison with their findings difficult. In our study, the time to reperfusion was less than $4 \mathrm{~h}$ after symptom onset in $89.3 \%$ of the patients, and the results are probably best compared with the following two studies, which both included patients with STEMI treated with primary PCI within $6 \mathrm{~h}$ of symptom onset. Mehta et al. reported in the APEX-AMI trial that $2.0 \%$ of patients developed VT or VF after cardiac catheterization [13]. Furthermore, Mehta et al. undertook an analysis of outcomes from the HORIZONS-AMI trial and reported that $5.2 \%$ of patients developed VT/VF after PCI [14]. Only a limited comparison is possible with the following studies because the time to primary PCI after symptom onset was longer in those studies. An analysis by Ohlow of an observational registry of patients with STEMI treated with primary PCI within $24 \mathrm{~h}$ of symptom onset revealed that the incidence of malignant arrhythmia was $4.7 \%$; however, the investigators did not state where the arrhythmias occurred, and they observed endpoints only during the $\mathrm{CCU}$ stay [12]. A single-centre retrospective cohort study of patients with STEMI treated with primary PCI within $24 \mathrm{~h}$ of symptom onset undertaken by Cricri and colleagues reported a comparable number of patients $(2.6 \%)$ who developed VT or VF after cardiac catheterization [11].

There are limited data on the potential therapeutic benefit of primary PCI compared to thrombolysis in terms of the incidence of in-hospital malignant arrhythmias in patients with acute STEMI. The incidence of malignant ventricular arrhythmia in our cohort was less than the VF or sustained VT incidence of $10.2 \%$ reported in the GUSTO-I study, a large randomized clinical trial investigating thrombolysis with streptokinase in patients with STEMI within $6 \mathrm{~h}$ of symptom onset [15]. This observation corroborates the hypothesis of PCI being superior to thrombolysis.

We observed a predominantly biphasic distribution of composite endpoint events, with $76.7 \%$ occurring within $96 \mathrm{~h}$ of symptom onset and $12.6 \%$ occurring between 240 and $360 \mathrm{~h}$. This biphasic pattern differs from the more monophasic distribution observed in the thrombolysis era as well as in studies of major arrhythmias after successful primary angioplasty for acute STEMI. In the GUSTO-1 thrombolysis trial, 39 and $55 \%$ of in-hospital deaths occurred within 24 and $48 \mathrm{~h}$ of randomization, respectively, while $84 \%$ of malignant arrhythmias occurred within $48 \mathrm{~h}$ of randomization [16]. In study settings similar to ours with patients treated within $6 \mathrm{~h}$ of symptom onset, Mehta and colleagues found, in retrospective analyses of the APEX-AMI study population and in the prospective HORIZONS-AMI trial, that 70 and $85 \%$, respectively, of VT-associated fatal events occurred within the first $48 \mathrm{~h}$ of leaving the catheterization laboratory $[13,14]$. In the two studies that included patients with STEMI treated with primary PCI within 24 h of symptom onset, a different temporal distribution was observed. In the study of Cricri and colleagues, most of the malignant arrhythmias (sustained VT, VF or bradycardia necessitating cardiac pacing) developed in the catheterization laboratory, and nearly all of these arrhythmias occurred within $24 \mathrm{~h}$ [11]. 
Ohlow and colleagues reported $90 \%$ of VTs occurring within the first $48 \mathrm{~h}$ [12].

Our secondary objective was to identify clinical predictors associated with the occurrence of the combined endpoint of VT, SCD or arrhythmic death, and resuscitation in the early phase of acute transmural infarction. These predictors would a) identify patients at high risk for the combined endpoint at the time of hospitalization and b) identify patients at risk despite the apparent lack of established risk factors, e.g., cardiogenic shock.

The variables used for our logistic regression modelling were based on observations from prior studies of risk stratification and include patient demographic and clinical characteristics, measures of the acuity and angiographic presentation of the MI, and indicators of the type and extent of myocardial ischaemia and necrosis $[4,5]$. Our results indicate that age, severely impaired LVEF, peak serum CK concentration, leucocytosis and the presence of coronary thrombus were positively associated with the combined endpoint. In patients treated by primary $\mathrm{PCI}$ in the APEX-AMI trial as well as those in the study of Ohlow and colleagues, a post-procedural TIMI flow of less than grade 3 was associated with VT or VF $[12,13]$. In the APEX-AMI trial, leucocytosis was also a predictor of ventricular arrhythmia [13]. A similar observation was also made by Rahimi et al. in patients with NSTEMI [17].

In several studies from the thrombolysis era, age, severely impaired LVEF and peak serum CK concentration have also been consistently associated with a higher incidence of VF or VT during or immediately after AMI. An analysis of the Holter Registry data from the Cardiac Arrhythmia Suppression Trials showed that age and reduced LVEF were independent predictors of the incidence and frequency of VT [18]. The analysis by Ruiz-Bailén and colleagues of the ARIAM Database also showed that age and peak CK concentration were associated with VF [19]. In the study by Mont and colleagues of patients with AMI who were referred to a CCU after thrombolysis, serum CK-MB fraction concentration, Killip class and bifascicular block were independent predictors of the development of sustained monomorphic tachycardia [20].

We also reported on secondary outcomes and found that in our 'real-world' single-centre registry of patients with acute transmural infarction treated with primary PCI, total in-hospital mortality was $6.5 \%$, which is consistent with other reports. In similarly designed retrospective single-centre studies by Giglioli et al. and Kozieradzka et al., in-hospital mortality was $5.9 \%$ and $30-d$ mortality was $6.3 \%[4,10]$. A more recent small single-centre study in China showed that mortality was $8.6 \%$ in patients aged $>60$ years compared with $1.5 \%$ in the non-elderly group [21]. In our cohort, $15 \%$ of patients with sustained VT and $93 \%$ of patients with VF died, resulting in a mortality rate among those who developed a ventricular arrhythmia twice that in a retrospective cohort study of 2317 patients with AMI reported by Henkel et al. (mortality rate $38 \%$ ) and the APEX-AMI study (mortality rate 33\%) [1, 13]. The incidence of bradyarrhythmia in our cohort was also lower than that in other reports; specifically, only $1.3 \%$ of the patients developed bradyarrhythmia (with $0.8 \%$ of the cases being $\mathrm{CHB}$ ), while Giglioli et al. reported an incidence of $6.3 \%$ [10].

\section{Possible mechanisms and explanations}

Our observation of a biphasic temporal distribution of the combined endpoint can be explained by the nature of our chosen endpoint, which comprised episodes of all major ventricular arrhythmias, SCD/AD and resuscitation not only during the initial phase when patients were continuously monitored in the $\mathrm{CCU}$ but also during the entire hospital stay. In addition, we did not consider any events that occurred before or during cardiac catheterization $[9,11]$. Other potential explanations are that the time to PCI was longer (up to $24 \mathrm{~h}$ ) in other studies $[11,12]$, and thus, myocardial necrosis may have been more pronounced in these studies. Furthermore, our population was unselected, unlike trials of study drugs/drug-eluting stents, such as the APEX-AMI and HORIZONS-AMI trials, in which some potential participants were excluded $[13,14]$.

The multivariate analysis identified variables associated with the composite endpoint that differed from those of other studies, potentially because our endpoint included all ventricular arrhythmias, SCD/AD and resuscitation episodes, while other studies used only ventricular arrhythmias and $\mathrm{CHB}$ as the endpoint. Furthermore, leucocytosis, elevated CK concentration and severely impaired LVEF would not have been a consequence of staged or advanced infarctions in our cohort, as we only included patients with $<12 \mathrm{~h}$ of symptoms in whom necrosis and reactive inflammation would not have become established. In addition, $92.4 \%$ of our patients underwent primary PCI within $4 \mathrm{~h}$ of symptom onset.

It is challenging to explain the relatively high mortality rate of those who developed arrhythmia in our cohort. We cannot conclude that adverse outcomes were due to cardiogenic shock and VF alone, as $>40 \%$ of the patients in our cohort who died after an episode of VF did not exhibit symptoms or signs of low cardiac output.

Barron et al. concluded in the retrospective analyses of a TIMI 10 thrombolysis study that an elevated WBC was associated with reduced epicardial blood flow and myocardial perfusion, thromboresistance (arteries open later and have a greater thrombus burden), and a higher incidence of new congestive heart failure and death [22]. Our data therefore seem to suggest that inflammation and the WBC itself may also be directly correlated with 
coronary thrombosis, impaired perfusion, and reperfusion injury in the PCI era.

\section{Study strengths and limitations}

Our cohort comprised 975 multi-ethnic patients with a clearly defined pathophysiological substrate (transmural AMI) and therapy (only primary PCI for reperfusion of the occluded vessel). In this cohort, we found a lower incidence of in-hospital major arrhythmia, SCD/AD and resuscitation but a higher mortality rate and biphasic temporal distribution of those who met the composite endpoint. Multivariable regression analysis showed positive associations between several factors and the combined endpoint. Due to the low number of events, we were unable to develop and calculate a risk score for the occurrence of the combined endpoint. The low number of events may be a consequence of the retrospective nature of this study.

A major concern may relate to the period in which data were collected (2005-2011) and the procedural aspects, i.e., the P2Y12 inhibition provided (Clopidogrel) and the very low percentage of implanted DES during primary PCI (12.6\%) in our study. In the EUROMAX trial, the choice of prasugrel or ticagrelor over clopidogrel was not associated with differences in acute stent thrombosis or 30-day ischaemic outcomes after PCI [23]. Furthermore, in the PRAGUE-18 study, prasugrel and ticagrelor were found to be similarly effective during the first year after MI, and economically motivated early post-discharge switches to clopidogrel were not associated with an increased risk of ischaemic events [24]. The low percentage of implanted DES (all first generation) in our study should not be of concern because a meta-analysis [25], cost analysis data [26], cohort registries [27] as well as single-centre studies [28] have shown that the only benefit of DES is the reduction of target vessel revascularization. Total mortality and MACE or stent thrombosis are not superior in patients with STEMI receiving bare metal stents (BMS), although a trend toward lower mortality may be seen with everolimus-eluting stents (EES) [29]. Furthermore, in the Norwegian Coronary Stent (NORSTENT) trial, patients (26\% with STEMI) were randomized to the DES or BMS group. There were no differences in the incidence of the primary endpoint (a composite of death from any cause or non-fatal spontaneous MI) after a median follow-up of 5 years [30].

Another limitation is the potential lack of generalizability of this single-centre study, which may not reflect outcomes achieved by other teams in different settings. Furthermore, observational studies may generate only a hypothesis that remains to be proven in a randomized controlled trial [31].

\section{Conclusions}

In this population of patients with acute transmural myocardial infarction treated with early PCI, we found that the total burden of malignant ventricular arrhythmias was reduced but that the mortality rate of those who developed these arrhythmias was substantially higher. Reperfusion by PCI resulted in a biphasic distribution of all major ventricular arrhythmias, SCD/AD or resuscitation. This result differed from the single peak pattern described in previous studies with thrombolysis as well as with primary PCI.

Nearly one-third of malignant arrhythmias and/or deaths occur late in the clinical course, and predictors are necessary to prevent such events. Due to the low numbers of events and the retrospective nature of this study, more evidence of the predictive value of the identified variables is needed before firm conclusions can be drawn. At this point, we would not recommend any change in clinical practice, as there is no basis for an algorithm to identify patients at risk. Our findings should, however, prompt a multi-centre study to examine the predictors that we have identified in greater detail, to establish a risk scoring system, and to investigate the influence of preventive measures, such as longer periods of monitoring and the use of wearable defibrillator vests.

\section{Abbreviations}

AD: Arrhythmic death; AMI: Acute myocardial infarction; BMS: Bare metal stent; CCU: Coronary care unit; CHB: Complete heart block; CK: Creatine

phosphokinase; DES: Drug-eluting stent; ECG: Electrocardiogram;

EES: Everolimus-eluting stent; EF: Ejection fraction; ICU: Intensive care unit; IRA: Infarct-related artery; LAD: Left anterior descending; LBBB: Left bundle branch block; LV: Left ventricular; LVEF: Left ventricular ejection fraction; M: Myocardial infarction; NSTEMI: Non-ST-elevation myocardial infarction; PAD: Peripheral artery disease; PCI: Percutaneous coronary interventions; PEA: Pulseless electric activity; SCD: Sudden cardiac death; STEMI: ST-elevation myocardial infarction; TIMI: Thrombolysis in myocardial infarction; VF: Ventricular fibrillation; VT: Ventricular tachycardia; WBC: White blood cell count

\section{Acknowledgements}

The authors thank Dunja Schulenburg and Dirk Böhm for their help with the collection of data, technical assistance and management of the databases.

\section{Funding}

This work was supported by a grant from the German Cardiac Society financed by GlaxoSmithKline.

\section{Availability of data and materials}

The datasets generated and/or analysed during the current study are not publicly available due to hospital policy but are available from the corresponding author upon reasonable request.

\section{Authors' contributions}

MA was responsible for the study conception and design, interpretation of the data, and drafting of the manuscript. PM and KA collected, analysed and interpreted the data. TO and SS were responsible for the statistical analysis and data interpretation. WS examined and revised the manuscript for intellectual content and gave final approval of the manuscript submitted. All authors read and approved the final version of the manuscript.

Ethics approval and consent to participate

Our study involved human participants and their data. The investigation was approved by the local hospital management. Our institution's ethics 
committee declared that no ethics approval or consent was necessary (Ärztekammer Nordrhein, Germany; reference number 19-2013).

\section{Competing interests}

The authors declare that they have no financial or non-financial competing interests regarding the research conducted.

\section{Publisher's Note}

Springer Nature remains neutral with regard to jurisdictional claims in published maps and institutional affiliations.

\section{Author details}

${ }^{1}$ Herzzentrum Duisburg, Gerrickstr. 21, D-47137 Duisburg, Germany. ${ }^{2}$ Stiftung Institut für Herzinfarktforschung, Bremserstraße 79 - Haus, MD-67063 Ludwigshafen a. Rh, Germany. ${ }^{3}$ Klinik für Kardiologie und Angiologie, Herzzentrum Duisburg, Gerrickstr. 21, 47137 Duisburg, Germany. ${ }^{4}$ Present address: Herzzentrum Hirslanden Zentralschweiz, Klinik St. Anna, St. Anna Str. 32, CH-6006 Luzern, Switzerland.

\section{Received: 20 September 2017 Accepted: 31 May 2018} Published online: 14 June 2018

\section{References}

1. Henkel DM, Witt BJ, Gersh BJ, Jacobsen SJ, Weston SA, Meverden RA, et al. Ventricular arrhythmias after acute myocardial infarction: a 20-year community study. Am Heart J. 2006:151:806-12.

2. Hasin Y, Danchin N, Filippatos GS, Heras M, Janssens U, Leor J, et al. Recommendations for the structure, organization, and operation of intensive cardiac care units. Eur Heart J. 2005:26:1676-82.

3. Kaul P, Newby LK, Fu Y, Mark DB, Califf RM, Topol EJ, et al. International differences in evolution of early discharge after acute myocardial infarction. Lancet. 2004;363:511-7.

4. Kozieradzka A, Kaminski KA, Maciorkowska D, Olszewska M, Dobrzycki S, Nowak K, et al. GRACE, TIMI, Zwolle and CADILLAC risk scores-do they predict 5-year outcomes after ST-elevation myocardial infarction treated invasively? Int J Cardiol. 2011;148:70-5.

5. TIMI Study Group. The thrombolysis in myocardial infarction (TIMI) trial. Phase I findings. N Engl J Med. 1985;312:932-6.

6. Keeley EC, Boura JA, Grines CL. Primary angioplasty versus intravenous thrombolytic therapy for acute myocardial infarction: a quantitative review of 23 randomised trials. Lancet. 2003;361:13-20.

7. Widimsky P, Budesinsky T, Vorac D, Groch L, Zelizko M, Aschermann M, et al. Long distance transport for primary angioplasty vs immediate thrombolysis in acute myocardial infarction. Final results of the randomized national multicentre trial-PRAGUE-2. Eur Heart J. 2003;24:94-104.

8. Busk M, Maeng M, Rasmussen K, Kelbaek H, Thayssen P, Abildgaard U, et al. The Danish multicentre randomized study of fibrinolytic therapy vs. primary angioplasty in acute myocardial infarction (the DANAMI-2 trial): outcome after 3 years follow-up. Eur Heart J. 2008;29:1259-66.

9. Giglioli C, Margheri M, Valente S, Comeglio M, Lazzeri C, Romano SM, et al. The incidence and timing of major arrhythmias following successful primary angioplasty for acute myocardial infarction. Ital Heart J. 2005;6:28-34.

10. Giglioli C, Margheri M, Valente S, Comeglio M, Lazzeri C, Chechi T, et al. Timing, setting and incidence of cardiovascular complications in patients with acute myocardial infarction submitted to primary percutaneous coronary intervention. Can J Cardiol. 2006;22:1047-52.

11. Cricri P, Trachsel L, Muller P, Wackerlin A, Reinhart W, Bonetti P. Incidence and time frame of life-threatening arrhythmias in patients with ST-segment elevation myocardial infarction undergoing primary percutaneous coronary intervention. Swiss Med Wkly. 2012;8:13604.

12. Ohlow M, Geller J, Richter S, Farah A, Muller S, Fuhrmann J, et al. Incidence and predictors of ventricular arrhythmias after ST-segment elevation myocardial infarction. Am J Emerg Med. 2012;30:580-6.

13. Mehta R, Starr A, Lopes R, Hochman J, Widimsky P, Pieper K, et al. Incidence of and outcomes associated with ventricular tachycardia or fibrillation in patients undergoing primary percutaneous coronary intervention. JAMA 2009;301:1779-89.

14. Mehta R, Yu J, Piccini J, Tcheng J, Farkouh M, Reiffel J, et al. Prognostic significance of postprocedural sustained ventricular tachycardia or fibrillation in patients undergoing primary percutaneous coronary intervention (from the HORIZONS-AMI trial). Am J Cardiol. 2012;109:805-12.
15. Newby K, Thompson T, Stebbins A, Topol E, Califf R, Natale A. Sustained ventricular arrhythmias in patients receiving thrombolytic therapy: incidence and outcomes. The GUSTO Investigators. Circ. 1998;98:2567-73.

16. Lee KL, Woodlief LH, Topol EJ, Weaver WD, Betriu A, Col J, et al. Predictors of 30-day mortality in the era of reperfusion for acute myocardial infarction. Results from an international trial of 41,021 patients. GUSTO-I investigators. Circ. 1995:91:1659-68.

17. Rahimi K, Watzlawek S, Thiele H, Secknus MA, Hayerizadeh BF, Niebauer J, et al. Incidence, time course, and predictors of early malignant ventricular arrhythmias after non-ST segment elevation myocardial infarction in patients with early invasive treatment. Eur Heart J. 2006:27:1706-11.

18. Josephson R, Papa L, Brooks M, Morris M, Akiyama T, Greene H. Effect of age on postmyocardial infarction ventricular arrhythmias (Holter registry data from CAST I and CAST II). Cardiovascular arrhythmia suppression trials. Am J Cardiol. 1995:76:710-3.

19. Ruiz-Bailen M, Aguayo de Hoyos E, Ruiz-Navarro S, Issa-Khozouz Z, ReinaToral A, Diaz-Castellanos MA, et al. Ventricular fibrillation in acute myocardial infarction in Spanish patients: results of the ARIAM database. Crit Care Med. 2003;31:2144-51.

20. Mont L, Cinca J, Blanch P, Blanco J, Figueras J, Brotons C, et al. Predisposing factors and prognostic value of sustained monomorphic ventricular tachycardia in the early phase of acute myocardial infarction. J Am Coll Cardiol. 1996;28:1670-6.

21. Su Y, Cai X, Geng H, Sheng H, Fan M, Pan M. In-hospital clinical outcomes of elderly patients ( $>/=60$ years) undergoing primary percutaneous coronary intervention. Int J Clin Exp Med. 2015:8:11244-51.

22. Barron H, Cannon C, Murphy S, Braunwald E, Gibson C. Association between white blood cell count, epicardial blood flow, myocardial perfusion, and clinical outcomes in the setting of acute myocardial infarction: a thrombolysis in myocardial infarction 10 substudy. Circ. 2000;102:2329-34.

23. Huber K, Ducroca G, Hamm CW, Van 't Hof A, Lapostolle F, Coste P, et al. Early clinical outcomes as a function of use of newer oral P2Y12 inhibitors versus clopidogrel in the EUROMAX trial. Open Heart. 2017:4:e000677.

24. Motovska Z, Hlinomaz O, Kala P, Hromadka M, Knot J, Varvarovsky I, et al. 1 -year outcomes of patients undergoing primary angioplasty for myocardial infarction treated with prasugrel versus ticagrelor. J Am Coll Cardiol. 2018; 71:371-81.

25. Wang L, Wang $H$, Dong $P$, Li Z, Wang Y, Duan N, et al. Long-term outcomes of drug-eluting versus bare-metal stent for ST-elevation myocardial infarction. Arq Bras Cardiol. 2014;102:529-38.

26. Stella SF, Gehling Bertoldi E, Polanczyk CA. Contemporary context of drugeluting stents in Brazil: a cost utility study. Med Decis Mak. 2016:36:1034-42.

27. Natsuaki M, Morimoto T, Furukawa Y, Nakagawa Y, Kadota K, Yamaji K, et al. Late adverse events after implantation of sirolimus-eluting stent and bare-metal stent: long-term (5-7 years) follow-up of the coronary revascularization demonstrating outcome study-Kyoto registry Cohort-2. Circ Cardiovasc Interv. 2014;7:168-79.

28. Lee WW, Cheng FW, Choi AY, Fong ST, Yu CM, Yan BP. Clinical, humanistic, and economic outcomes between drug-eluting stent (DES) and bare metal stent (BMS): 18-month follow-up study. J Med Econ. 2017;20:239-45.

29. Iqbal MB, Nadra IJ, Ding L, Fung A, Aymong E, Chan AW, et al. Long-term outcomes following drug-eluting stents versus bare metal stents for primary percutaneous coronary intervention: a real-world analysis of 11,181 patients from the British Columbia cardiac registry. Catheter Cardiovasc Interv. 2016; 88:24-35.

30. Bonaa KH, Mannsverk J, Wiseth R, Aaberge L, Myreng Y, Nygard O, et al. Drug-eluting or bare-metal stents for coronary artery disease. N Engl J Med. 2016:375:1242-52

31. Ziff OJ, Lane DA, Samra M, Griffith M, Kirchhof P, Lip GY, et al. Safety and efficacy of digoxin: systematic review and meta-analysis of observational and controlled trial data. BMJ. 2015;351:h4451. 